\title{
Echocardiographic changes with non-invasive ventilation and CPAP in obesity hypoventilation syndrome
}

\author{
Jaime Corral, ${ }^{1,2}$ Maria Victoria Mogollon, ${ }^{3}$ M-Ángeles Sánchez-Quiroga, ${ }_{1}^{2,4}$ \\ Javier Gómez de Terreros, ${ }^{1,2}$ Auxiliadora Romero, ${ }^{5}$ Candela Caballero, ${ }^{5}$ \\ Joaquin Teran-Santos, ${ }^{2,6}$ María L Alonso-Álvarez, ${ }^{2,6}$ Teresa Gómez-García, ${ }^{7}$ \\ Mónica González, ${ }^{8}$ Soledad López-Martínez ${ }^{9}$ Pilar de Lucas, ${ }_{1}^{9}$ José M Marin ${ }^{2,10}$ \\ Odile Romero, ${ }^{2,11}$ Trinidad Díaz-Cambriles, ${ }^{2,12}$ Eusebi Chiner, ${ }^{13}$ Carlos Egea, ${ }^{2,14}$ \\ Roberto M Lang, ${ }^{15}$ Babak Mokhlesi, $^{16}$ Juan F Masa, ${ }^{1,2}$ on behalf of The Spanish Sleep \\ Network
}

- Additional material is published online only. To view please visit the journal online (http://dx.doi.org/10.1136/ thoraxjnl-2017-210642).

For numbered affiliations see end of article.

\section{Correspondence to}

Dr Juan F Masa, Calle Rafael Alberti 12, 10005 Cáceres, spain; fmasa@separ.es

Received 14 June 2017 Revised 20 September 2017 Accepted 30 October 2017 Published Online First 16 November 2017
Check for updates

To cite: Corral J Mogollon MV, SánchezQuiroga M-Á, et al. Thorax 2018:73:361-368.

\section{ABSTRACT}

Rationale Despite a significant association between obesity hypoventilation syndrome (OHS) and cardiac dysfunction, no randomised trials have assessed the impact of non-invasive ventilation (NIV) or CPAP on cardiac structure and function assessed by echocardiography.

Objectives We performed a secondary analysis of the data from the largest multicentre randomised controlled trial of OHS (Pickwick project, $n=221$ ) to determine the comparative efficacy of 2 months of NIV $(n=71)$, CPAP $(n=80)$ and lifestyle modification (control group, $n=70$ ) on structural and functional echocardiographic changes.

Methods Conventional transthoracic two-dimensional and Doppler echocardiograms were obtained at baseline and after 2 months. Echocardiographers at each site were blinded to the treatment arms. Statistical analysis was performed using intention-to-treat analysis.

Results At baseline, $55 \%$ of patients had pulmonary hypertension and $51 \%$ had evidence of left ventricular hypertrophy. Treatment with NIV, but not CPAP, lowered systolic pulmonary artery pressure $(-3.4 \mathrm{~mm} \mathrm{Hg}$, $95 \% \mathrm{Cl}-5.3$ to -1.5 ; adjusted $\mathrm{P}=0.025$ vs control and $\mathrm{P}=0.033 \mathrm{vs}$ (PAP). The degree of improvement in systolic pulmonary artery pressure was greater in patients treated with NIV who had pulmonary hypertension at baseline ( $-6.4 \mathrm{~mm} \mathrm{Hg}, 95 \% \mathrm{Cl}-9$ to -3.8$)$. Only NIV therapy decreased left ventricular hypertrophy with a significant reduction in left ventricular mass index $\left(-5.7 \mathrm{~g} / \mathrm{m}^{2}\right.$; $95 \% \mathrm{Cl}-11.0$ to -4.4 ). After adjusted analysis, NIV was superior to control group in improving left ventricular mass index $(P=0.015)$. Only treatment with NIV led to a significant improvement in 6 min walk distance ( $32 \mathrm{~m}$; 95\% Cl 19 to 46).

Conclusion In patients with OHS, medium-term treatment with NIV is more effective than CPAP and lifestyle modification in improving pulmonary hypertension, left ventricular hypertrophy and functional outcomes. Long-term studies are needed to confirm these results.

Trial registration number Pre-results, NCT01405976 (https://clinicaltrials.gov/).
Key messages

What is the key question?

- No randomised trials have assessed the impact of non-invasive ventilation (NIV) or CPAP on cardiac structure and function assessed by echocardiography in patients with obesity hypoventilation syndrome (OHS).

What is the bottom line?

- Both NIV and CPAP improve clinical symptoms, polysomnographic parameters and daytime $\mathrm{PaCO}_{2}$ in OHS. Whether NIV is more effective than CPAP in improving the cardiovascular burden imposed by OHS requires further investigation.

Why read on?

- This is the first randomised controlled trial that evaluates changes in cardiac structure and function with NIV, CPAP and control. NIV, but not CPAP, improves pulmonary hypertension and left ventricular hypertrophy.

\section{INTRODUCTION}

Obesity hypoventilation syndrome (OHS) is defined by a combination of obesity, daytime hypercapnia and sleep-disordered breathing in the absence of an alternative neuromuscular, mechanical or metabolic explanation for hypoventilation. Approximately 90\% of patients with OHS have concomitant obstructive sleep apnoea (OSA), with $73 \%$ having severe OSA. ${ }^{1}$ Although the exact prevalence of OHS in the community is unknown, it is likely to increase in parallel with the rise in severe obesity (body mass index or BMI $\geq 40 \mathrm{~kg} / \mathrm{m}^{2}$ and $\geq 50 \mathrm{~kg} / \mathrm{m}^{2}$ ). ${ }^{2}$ In fact, the prevalence of OHS among patients with OSA and a BMI $\geq 40 \mathrm{~kg} / \mathrm{m}^{2}$ is alarmingly high at $20 \%-30 \%$. $^{3}$

Positive airway pressure (PAP) therapy is the cornerstone intervention in patients with OHS. PAP therapy can be administered as non-invasive ventilation (NIV) or as CPAP. Both NIV and CPAP improve 


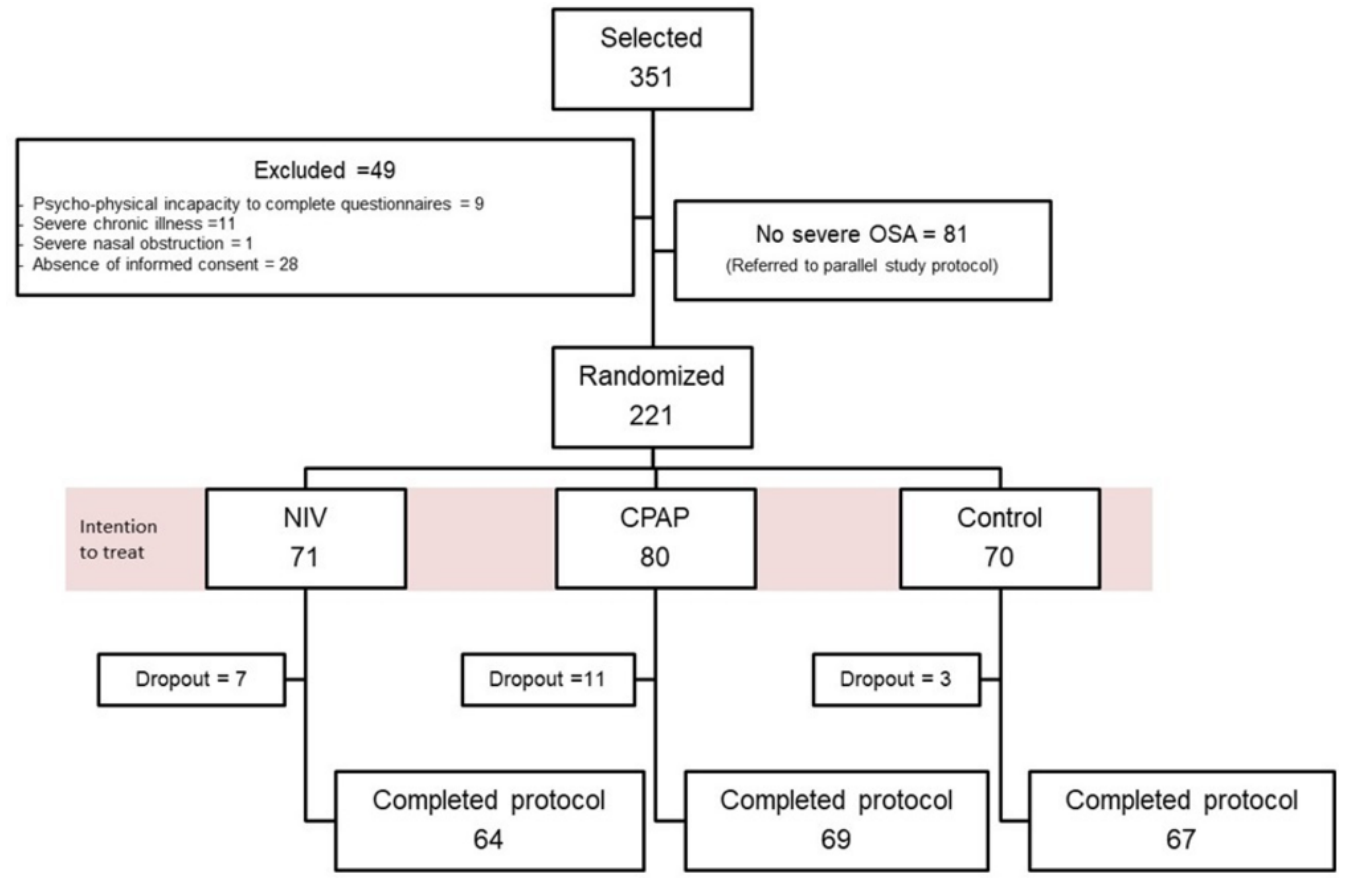

Figure 1 Flow chart of the study protocol. Of the 351 selected patients, 49 were excluded and 221 with severe OSA (AHI $\geq 30)$ were randomised to NIV, CPAP or control groups. Eighty-one patients without severe OSA were referred to a parallel randomised controlled trial. AHI, Apnoea Hypopnoea Index; NIV, non-invasive ventilation; OSA, obstructive sleep apnoea.

daytime symptoms, quality of life, sleep quality, and daytime and nocturnal gas exchange. ${ }^{14-7}$ Moreover, PAP therapy lowers intrathoracic pressure swings and sympathetic activity. ${ }^{8}$ Several randomised controlled trials have shown clinical, spirometric and polysomnographic improvements with both PAP treatment modalities. ${ }^{1457}$ However, there may be some respiratory functional advantages with NIV. ${ }^{1}$

Compared with patients with eucapnic OSA, patients with OHS have a higher prevalence of cardiovascular morbidity, hypertension, diabetes and dyslipidaemia. ${ }^{9-11}$ Moreover, the high mortality observed in patients with untreated OHS has been partly attributed to cardiovascular morbidity. ${ }^{12}$ Although a few small observational studies have reported a high prevalence of pulmonary hypertension and right ventricular dysfunction in patients with OHS, ${ }^{13-17}$ randomised controlled trials assessing the impact of NIV or CPAP on cardiac outcomes have not been performed.

Given the very limited information on the effect of PAP therapy on cardiac function and structure in patients with OHS, we aimed to assess the impact of the two most commonly prescribed PAP treatment modalities in OHS (ie, NIV and CPAP) on commonly reported echocardiographic parameters as a hypothesis generating model. We analysed data from the largest multicentre randomised controlled trial with three open parallel groups. This study was designed to determine the comparative efficacy of NIV, CPAP and lifestyle modification (control group) after 2 months of follow-up (Pickwick Study) including a priori echocardiographic assessments as a secondary objective. ${ }^{16} 18$

\section{METHODS}

\section{Patients}

From May 2009 to March 2013, we screened consecutive patients between 15 years and 80 years old who were referred for pulmonary consultation for suspected OHS or OSA in 16 tertiary care hospitals in Spain. For the initial medium-term outcomes of this study (see online supplementary material) we enrolled subjects who had OHS with severe OSA (Apnoea Hypopnoea Index $\geq 30$ events/h). ${ }^{1}$ OHS was defined as (1) obesity with $\mathrm{BMI} \geq 30 \mathrm{~kg} / \mathrm{m}^{2}$ plus stable hypercapnic respiratory failure during wakefulness $\left(\mathrm{PaCO}_{2} \geq 45 \mathrm{~mm} \mathrm{Hg}, \mathrm{pH} \geq 7.35\right)$, (2) no clinical worsening during the previous 2 months and (3) no evidence of relevant $\mathrm{COPD}\left(\mathrm{FEV}_{1}>70 \%\right.$ of predicted when $\mathrm{FEV}_{1} /$ (FVC >70), neuromuscular, chest wall or metabolic disease. Other inclusion criteria were an absence of narcolepsy or restless legs syndrome, and a correctly executed $30 \mathrm{~min}$ CPAP/NIV treatment test (see online supplementary material). The exclusion criteria were: (1) a psychophysical inability to complete questionnaires, (2) severe chronic debilitating illness, (3) severe chronic nasal obstruction and (4) lack of informed consent. The study was approved by the ethics committees of the 16 centres and written informed consent was obtained from all patients.

\section{Interventions}

All participants underwent conventional inlaboratory polysomnography (PSG) and an analysis of arterial blood gases (ABGs). All patients were ambulatory and were randomised by an electronic database (simple randomisation) into the NIV, CPAP or control groups (figure 1).

\section{Control group: lifestyle modification}

The lifestyle modification consisted of a 1000-calorie diet and maintenance of adequate sleep hygiene (see online supplementary material). Oxygen therapy was added if baseline daytime or nocturnal hypoxaemia was detected. ${ }^{18}$

\section{CPAP}

In addition to lifestyle modification and oxygen (if required), ${ }^{18}$ patients were instructed to use home CPAP therapy at a fixed pressure during the entire sleep period based on the results of 
a conventional CPAP titration study (see online supplementary material).

\section{Non-invasive ventilation}

In addition to lifestyle modification and oxygen (if required), ${ }^{18}$ patients were instructed to use NIV treatment during the entire sleep period. The ventilator mode was set at a bilevel PAP with assured volume. More information about the setting, daytime and polysomnographic adjustments, ventilators and masks used are available in the online supplementary material.

\section{Follow-up and outcomes}

At baseline we evaluated: ABG while breathing room air (see online supplementary material), anthropometric data, comorbidities, smoking and alcohol intake habits, level of dyspnoea (defined as a score of $\geq 2$ points in the Medical Research Council Scale), 6 min walk distance (6-MWD), PSG and conventional transthoracic echocardiogram. At 2 months, we evaluated: ABG while breathing room air, anthropometric data, level of dyspnoea, 6-MWD, Epworth Sleepiness Scale (ESS), blood pressure, PSG, spirometry and conventional transthoracic echocardiogram. Objective adherence to NIV or CPAP was also assessed at 2 months. Dropout definition is described in the online supplementary material.

\section{Polysomnography}

We performed PSG at baseline, for PAP titration (only for the NIV and CPAP groups), and after 2 months of treatment (with NIV and CPAP in place and without treatment for the control group) using standard protocols (see online supplementary material). Oxygen treatment was not applied during any of the polysomnographic studies.

\section{Echocardiogram}

Echocardiographers at each centre were blinded to the allocated treatment arm. All two-dimensional (2D) and Doppler echocardiograms were recorded using available echocardiographic equipment in each centre (see online supplementary material). Left ventricle (LV) size and wall thickness were measured according to European guidelines. ${ }^{19}$ All these parameters were derived from 2D-guided M-mode imaging or from linear measurements obtained from 2D images. LV ejection fraction (LVEF) was calculated from end-diastolic and end-systolic volume estimates, using volumetric measurements. LV mass was estimated by linear method with Cube's formula $(0.8 \times 1.04 \times$ $(($ IVS + LVID + PWT $) 3-$ LVID3 $)+0.6 \mathrm{~g})$, where LVID is the internal dimension of LV, PWT is the posterior wall thickness and IVS is the interventricular septum thickness, all measured at end-diastole in the parasternal long-axis view using either 2D-guided M-mode or linear measurements from 2D echocardiographic images. LV mass index was obtained dividing the LV mass by the body surface area according to Du Bois's formula. To assess LV diastolic function, we obtained peak early (E), peak atrial (A) mitral valve inflow velocities, as well as their ratio (E/A), isovolumic relaxation time (IVRT), deceleration time (DT) and the anteroposterior diameter of the left atrium measured in the parasternal long-axis view. Pulmonary artery systolic pressure was assessed from the maximum velocity of tricuspid regurgitation signal (continuous wave Doppler) by the addition of right atrial pressure, estimated from inferior cava vein and its collapsibility. Right ventricular systolic function was evaluated using the right ventricle index of myocardial performance (TEI Index). We considered a systolic pulmonary artery pressure $\geq 40 \mathrm{~mm} \mathrm{Hg}$ as evidence of pulmonary hypertension. LV hypertrophy was present if the LV mass index was $\geq 115 \mathrm{~g} / \mathrm{m}^{2}$ in men and $\geq 95 \mathrm{~g} / \mathrm{m}^{2}$ in women. ${ }^{20}$

\section{Statistical analysis}

Intention-to-treat analysis was performed. Missing values (dropouts included) were imputed following a multiple imputation method with iterative multivariable regression because the missing data had characteristics compatible with a missing at random pattern. We compared baseline characteristics of patients with OHS randomised to NIV, CPAP or control by analysis of variance (ANOVA) or using the $x^{2}$ test for categorical variables; if the overall significance was $<0.05$, we then performed comparison by pairs. Intragroup changes from baseline to 2 months were assessed using paired $t$ tests or equivalent non-parametrical test for continuous variables. The observed effects in the three arms of the study (intergroup differences) were compared using ANOVA. When the overall comparison was statistically significant $(p<0.05)$, paired comparisons of groups were performed by analysis of covariance (ANCOVA) taking into account the baseline value of the variable analysed, age, gender, BMI, presence of hypertension and diabetes, systolic and diastolic blood pressures and Oxygen Desaturation Index (henceforth 'basic adjustment').

In the NIV group we further analysed the change in systolic pulmonary artery pressure and 6-MWD according to the presence of pulmonary hypertension at baseline (systolic pulmonary artery pressure $\geq 40 \mathrm{~mm} \mathrm{Hg}$ ) using $t$ test. We also compared the change in LV mass index and 6-MWD according to the presence of LV hypertrophy at baseline (LV mass index $\geq 115 \mathrm{~g} / \mathrm{m}^{2}$ male, $\geq 95 \mathrm{~g} / \mathrm{m}^{2}$ female). In the NIV group we also compared the percentage of patients with pulmonary hypertension and LV hypertrophy at baseline and after 2 months of therapy using the $\mathrm{x}^{2}$ test. Correlations among changes with treatment in systolic pulmonary artery pressure, LV mass index and anthropometric, clinical and respiratory functional variables were carried out by Pearson's test.

Data management and statistical analyses were performed using SPSS software V.22.0 (IBM SPSS Statistics, Armonk, New York, USA).

\section{RESULTS}

Of the 351 patients who met inclusion criteria, 49 were excluded and 81 had mild-moderate OSA (figure 1). Dropout rates were similar in patients randomised to NIV, CPAP or lifestyle modification. Table 1 summarises baseline patient characteristics according to treatment allocation $(n=221)$. Patients were of middle age and generally severely obese, and there was a slight female preponderance. Comorbidities such as hypertension, diabetes and cardiovascular disorders were equally prevalent among the three groups. Patients randomised to CPAP were slightly younger than patients randomised to NIV $(p<0.01)$.

Due to technical difficulties, some echocardiographic variables were unavailable in $11 \%$ of the cohort. As described in the methods, missing values were imputed. Table 2 describes baseline echocardiographic measurements and the prevalence of abnormal echocardiographic parameters. ${ }^{20}$ The prevalence of structural and functional abnormalities was high across all subjects: $55 \%$ of patients had high systolic pulmonary artery pressure ( $\geq 40 \mathrm{~mm} \mathrm{Hg}$ ) and $51 \%$ had evidence of LV hypertrophy based on an LV mass index of $\geq 115 \mathrm{~g} / \mathrm{m}^{2}$ in men and $\geq 95 \mathrm{~g} / \mathrm{m}^{2}$ in women.

Table 3 summarises baseline and echocardiographic changes according to treatment allocation. Compared with the control 
Table 1 Baseline patient characteristics according to treatment allocation

\begin{tabular}{|c|c|c|c|}
\hline & $\begin{array}{l}\text { NIV } \\
n=71\end{array}$ & $\begin{array}{l}\text { CPAP } \\
n=80\end{array}$ & $\begin{array}{l}\text { Control } \\
n=70\end{array}$ \\
\hline Gender, male, \% & 35 & 52 & 44 \\
\hline Age, years, X (SD) & $64(11)$ & $57(13)^{*}$ & $60(13)$ \\
\hline $\mathrm{BMI}, \mathrm{kg} / \mathrm{m}^{2}, \mathrm{X}(\mathrm{SD})$ & $43(6.7)$ & $45(7.6)$ & $44(7)$ \\
\hline Active drinker, \% & 21 & 13 & 23 \\
\hline Active smoker, \% & 17 & 29 & 27 \\
\hline ESS, X (SD) & $11(5.1)$ & $11(4.8)$ & $11(5.3)$ \\
\hline Dyspnoea MRC Scale $\geq 2, \%$ & 48 & 37 & 56 \\
\hline Hypertension, \% & 76 & 65 & 62 \\
\hline $\mathrm{SBP}, \mathrm{mm} \mathrm{Hg}, \mathrm{X}(\mathrm{SD})$ & $136(15)$ & $140(17)$ & $136(16)$ \\
\hline DBP, mm Hg, X (SD) & $77(12)$ & $80(12)$ & $79(12)$ \\
\hline Diabetes, \% & 41 & 33 & 37 \\
\hline Dyslipidaemia, \% & 49 & 40 & 41 \\
\hline Ischaemic heart disease, \% & 8.5 & 11 & 10 \\
\hline Arrhythmia, \% & 13 & 2.5 & 10 \\
\hline Chronic heart failure, $\%$ & 18 & 13 & 16 \\
\hline Stroke, \% & 5.6 & 6.3 & 13 \\
\hline Leg arteriopathy, \% & 4.2 & 3.8 & 10 \\
\hline Pulmonary hypertension, $\%^{\dagger}$ & 7 & 6.3 & 13 \\
\hline At least one CVM, \% & 34 & 31 & 39 \\
\hline $\mathrm{pH}, \mathrm{X}(\mathrm{SD})$ & $7.41(0.03)$ & $7.40(0.04)$ & $7.39(0.04)$ \\
\hline $\mathrm{PaO}_{2}, \mathrm{~mm} \mathrm{Hg}, \mathrm{X}(\mathrm{SD})$ & $62(8.7)$ & $63(9.8)$ & $61(8.2)$ \\
\hline $\mathrm{PaCO}_{2}, \mathrm{~mm} \mathrm{Hg}, \mathrm{X}(\mathrm{SD})$ & $51(4.3)$ & $50(4.5)$ & $51(4.2)$ \\
\hline Bicarbonate, mmol/l, X (SD) & $30(3.4)$ & $30(4)$ & $30(3.2)$ \\
\hline $\mathrm{FEV}_{1}, \%, \mathrm{X}(\mathrm{SD})$ & $76(17)$ & $79(20)$ & $80(20)$ \\
\hline FVC, \%, X (SD) & $78(19)$ & $80(20)$ & $82(20)$ \\
\hline 6-MWD, metres, X (SD) & $340(132)$ & $358(131)$ & $338(112)$ \\
\hline Sleep efficiency, \%, X (SD) & $71(15)$ & $72(16)$ & $70(17)$ \\
\hline Light sleep, \%, X (SD) & $82(14)$ & $82(13)$ & $83(12)$ \\
\hline Deep sleep, \%, X (SD) & $8.5(10.0)$ & $9.2(10.2)$ & $8.5(9.2)$ \\
\hline REM sleep, \%, X (SD) & $9.1(6.8)$ & $9.3(8.0)$ & $8.6(6.2)$ \\
\hline Arousal index, X (SD) & $54(29)$ & $61(36)$ & $58(29)$ \\
\hline $\mathrm{AHI}, \mathrm{X}(\mathrm{SD})$ & $68(29)$ & $71(30)$ & $69(30)$ \\
\hline ODI, X (SD) & $63(31)$ & $72(35)$ & $68(31)$ \\
\hline $\mathrm{SpO}_{2}, \%, \mathrm{X}(\mathrm{SD})$ & $85(5.8)$ & $85(6.4)$ & $84(6.5)$ \\
\hline $\mathrm{SpO}_{2}<90 \%, \mathrm{X}(\mathrm{SD})$ & $69(29)$ & $67(30)$ & $72(26)$ \\
\hline Oxygen therapy, \% & 24 & 20 & 29 \\
\hline Compliance, hours/day & $5.3(2.3)$ & $5.3(2.1)$ & -- \\
\hline
\end{tabular}

${ }^{*} \mathrm{P}<0.05 \mathrm{NIV}$ versus CPAP

$\dagger=$ diagnosis prior to study entry

AHI, Apnoea Hypopnoea Index; BMI, body mass index; CVM,

cardiovascular morbidity; DBP, diastolic blood pressure; ESS, Epworth Sleepiness

Scale; MRC, Medical Research Council; NIV, non-invasive ventilation; ODI,

$3 \%$ Oxygen Desaturation Index; $\mathrm{PaCO}_{2}$, arterial pressure of $\mathrm{CO}_{2} ; \mathrm{PaO}_{2}$, oxygen

arterial pressure; REM, rapid eyes movement; SBP, systolic blood pressure;

$\mathrm{SpO}_{2}$, oxygen saturation by pulse oximetry; $\mathrm{X}$, mean; 6-MWD, 6 min walk distance.

group, NIV significantly lowered systolic pulmonary artery pressure and decreased LV hypertrophy (ie, septum thickness, LV posterior wall, LV mass and LV mass index) and these differences remained significant despite basic adjustment. Moreover, NIV led to a significant reduction in systolic pulmonary artery pressure
Table 2 Baseline echocardiographic measurements and prevalence of abnormal echocardiographic parameters

\begin{tabular}{|c|c|}
\hline & Mean $(S D)(n=221)$ \\
\hline Systolic PAP, mm Hg & $41(12)$ \\
\hline $\mathrm{PAP} \geq 40 \mathrm{~mm} \mathrm{Hg}, \%$ & 55 \\
\hline TEI Index & $0.41(0.12)$ \\
\hline TEl Index $\geq 0.43, \%$ & 43 \\
\hline EA ratio & $0.93(0.34)$ \\
\hline EA ratio $\leq 1, \%$ & 64 \\
\hline IVRT, ms & $97(29)$ \\
\hline IVRT $\geq 89 \mathrm{~ms}, \%$ & 61 \\
\hline DT, ms & $220(64)$ \\
\hline DT $\geq 220 \mathrm{~ms}, \%$ & 49 \\
\hline LAD, mm & $40(10)$ \\
\hline LAD $\geq 40 \mathrm{~mm}$ male, $\geq 38 \mathrm{~mm}$ female, $\%$ & 68 \\
\hline LVTDD, mm & $49(7.4)$ \\
\hline LVTDD $\geq 59 \mathrm{~mm}$ male, $\geq 53 \mathrm{~mm}$ female, $\%$ & 14 \\
\hline LVTSD, mm & $31(7.2)$ \\
\hline LVTSD $\geq 40 \mathrm{~mm}$ male, $\geq 35 \mathrm{~mm}$ female, $\%$ & 16 \\
\hline LVEF, \% & $64(9)$ \\
\hline LVEF $\leq 53, \%$ & 7.2 \\
\hline LV septum, mm & $13(2.4)$ \\
\hline LV septum $\geq 9 \mathrm{~mm}, \%$ & 95 \\
\hline LVPW, mm & $12(2.2)$ \\
\hline LVPW $\geq 9 \mathrm{~mm}, \%$ & 92 \\
\hline LV mass, gr & $224(64)$ \\
\hline LV mass $\geq 224 \mathrm{~g}$ male, $\geq 164 \mathrm{~g}$ female, $\%$ & 69 \\
\hline LV mass index, $\mathrm{g} / \mathrm{m}^{2}$ & $106(31)$ \\
\hline LV mass index $\geq 115 \mathrm{~g} / \mathrm{m}^{2}$ male, $\geq 95 \mathrm{~g} / \mathrm{m}^{2}$ female, $\%$ & 51 \\
\hline
\end{tabular}

LV, left ventricle; DT, deceleration time; EA, E and A waves; IVRT, isovolumic relaxation time; LAD, left atrial diameter; LVTSD, left ventricular telesystolic diameter; LVTDD, left ventricular telediastolic diameter; LVEF, left ventricular ejection fraction; LVPW, left ventricular posterior wall; PAP, pulmonary arterail pressure; TEI, global myocardial function.

and septum thickness when compared with CPAP in adjusted analysis. CPAP, however, reduced the DT when compared with controls without other echocardiographic benefits. Table s1 summarises the changes in $\mathrm{ABG}$, spirometry, 6-MWD, weight, blood pressure, ESS and polysomnographic parameters. NIV and CPAP treatment were more effective than lifestyle modification (control group) in improving ESS and polysomnographic parameters. NIV, however, was more effective than CPAP in improving $\mathrm{FEV}_{1}$ and 6-MWD. There were no significant intergroup differences in the degree of weight loss (see online supplementary material).

Figure s2 illustrates improvement of the systolic pulmonary artery pressure and 6-MWD in the NIV group according to the presence of pulmonary hypertension at baseline. The improvement in both parameters occurred mainly in the subgroup of patients with pulmonary hypertension at baseline. Figure s2 also shows the improvement in LV mass index and 6-MWD in the NIV group according to the presence of LV hypertrophy at baseline. The improvement in LV mass index and 6-MWD in the NIV group was not statistically different between those with or without LV hypertrophy at baseline. In the NIV group, the prevalence of pulmonary hypertension was reduced from $62 \%$ to $42 \%$ 
Table 3 Baseline changes in echocardiographic parameters based on treatment allocation

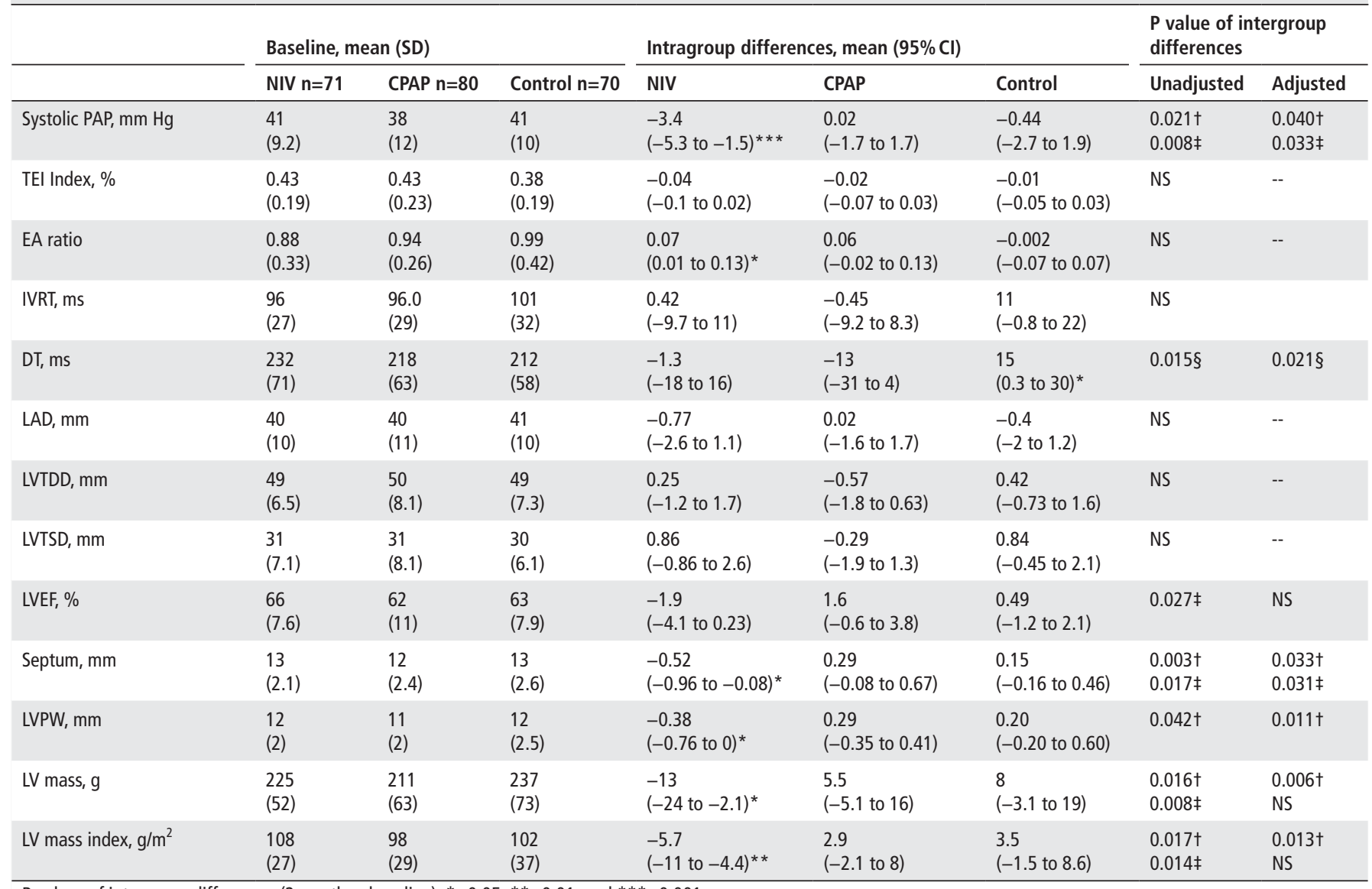

P values of intragroup differences ( 2 months - baseline): ${ }^{*}<0.05{ }^{* *}<0.01$; and ${ }^{* * *}<0.001$

$P$ values of intergroup differences unadjusted or adjusted by basic adjustment (baseline values of the variable analysed and age, gender, BMI, systolic and diastolic blood pressures, hypertension, diabetes presence and Oxygen Desaturation Index). † NIV and control; ₹ NIV and CPAP and § CPAP and control.

LV, left ventricle; DT, deceleration time; EA, E and A waves; IVRT, isovolumic relaxation time; LAD, left atrial diameter; LVTSD, left ventricular telesystolic diameter; LVTDD,

left ventricular telediastolic diameter; LVEF, left ventricular ejection fraction; LVPW, left ventricular posterior wall; NIV, non-invasive ventilation; NS, non significant; PAP, pulmonary arterail pressure; TEl, global myocardial function.

$(\mathrm{p}<0.01)$ and the prevalence of LV hypertrophy from $61 \%$ to $53 \%$ (non significant). Table s2 provides the correlation among changes with treatment in systolic pulmonary artery pressure, LV mass index and anthropometric, polysomnographic, clinical and respiratory functional variables included in table s1. Improvement in systolic pulmonary artery pressure was associated with improvement in $\mathrm{PaCO}_{2}$ and $\mathrm{FEV}_{1}$ while the improvement in $\mathrm{LV}$ mass index was associated with improvement in 6-MWD.

\section{DISCUSSION}

The Pickwick Study is the largest randomised clinical trial to date comparing NIV, CPAP and lifestyle modification in patients with OHS. Moreover, our study is the only clinical trial that systematically compared the effect of the abovementioned interventions on structural and functional echocardiographic changes. The main results can be summarised as follows: (1) more than half of the patients with OHS had echocardiographic evidence of pulmonary hypertension and LV hypertrophy; (2) NIV was more effective in improving LV hypertrophy parameters compared with control; and (3) only NIV therapy led to a significant reduction in pulmonary artery pressure. Only patients treated with NIV experienced a significant improvement in their 6-MWD.

Pulmonary hypertension is the most recognised haemodynamic disorder in patients with OHS but its prevalence has varied in different studies, likely due different patient populations and methods of measurement. ${ }^{13-17}$ Notably, only 19 out of 221 patients $(8.5 \%)$ in our cohort were diagnosed with pulmonary hypertension prior to enrolment, suggesting a significant underdiagnosis of this important comorbidity. The high prevalence of pulmonary hypertension observed in our cohort is in line with prior studies using similar echocardiographic criteria. ${ }^{13} 17$ In contrast, the prevalence LV hypertrophy was higher in our cohort than prior studies. In a small case series of 30 patients with OHS, Castro-Añon et al did not find any evidence of LV hypertrophy. ${ }^{13}$ In a case-control study of 50 patients with OHS and 50 matched obese controls, Russo et al reported a higher degree of LV diastolic dysfunction measured by the E/A ratio compared with matched obese controls. ${ }^{21}$ Notably, the E/A ratio in their OHS group were similar to the present study.

In a small clinical series of 30 patients with OHS who were treated with 6 months of NIV, the LV mass decreased by $12.3 \mathrm{~g}$. However, this clinically significant reduction in LV mass was not statistically significant, likely due to the small sample size. ${ }^{13}$ In the current study, we report a significant reduction in LV mass after 2 months of NIV therapy $(-13 \mathrm{~g})$. This degree of reduction in LV mass was accompanied by a significant reduction in LV mass index $\left(5.7 \mathrm{~g} / \mathrm{m}^{2}\right)$. This is clinically relevant given that LV hypertrophy as measured by echocardiogram is a marker of cardiac end-organ damage and an established surrogate end point for cardiovascular morbidity and mortality. ${ }^{22}$ Moreover, 
reduction of LV hypertrophy has been associated with a lower incidence of cardiovascular events in patients with hypertension. The reduction in LV mass index observed with NIV therapy in our patients with OHS is of similar magnitude to that observed after 3 months of cardiac resynchronisation therapy ${ }^{23}$ or after 9 months of therapy with a direct renin inhibitor, an angiotensin-receptor blocker or their combination in patients with hypertension, ${ }^{24}$ or after 12 months of spironolactone in patients with heart failure and preserved ejection fraction. ${ }^{25}$ Moreover, the degree of improvement in the LV mass index with NIV therapy was similar to what has been achieved with $\beta$-blockers. ${ }^{26}$ It is important to point out that the improvement in LV hypertrophy with NIV was not accompanied by an improvement in daytime blood pressure. Although we did not measure blood pressure during sleep, it is plausible that by being more effective in treating nocturnal hypoventilation, NIV led to better blood pressure control during sleep compared with CPAP and the control group. In contrast to CPAP, improvement in lung volumes with NIV can lead to lower intrathoracic pressure swings during sleep which can further reduce LV afterload. Notably, LV hypertrophy confers an excess risk for cerebrovascular events independently of blood pressure. ${ }^{27} 28$ Therefore, a reduction in LV hypertrophy without a significant change in blood pressure can be considered a clinically relevant outcome. In our cohort, the change in the 6-MWD correlated with the improvement of LV mass index which may be an expression of its clinical significance. However, it remains unclear whether improvements in exercise capacity leads to improvement in LV mass index or vice versa.

NIV therapy also led to a reduction in systolic pulmonary artery pressure. Patients with OHS experience pronounced intermittent hypoxaemia and swings in intrathoracic pressures due to frequent upper airway obstruction. These phenomena may promote a more robust degree of pulmonary vasoconstriction and vascular endothelial remodelling which could ultimately lead to the development of pulmonary hypertension. ${ }^{29}$ Although several relatively small studies have reported a reduction in pulmonary artery pressure ${ }^{3031}$ in eucapnic patients with OSA treated with 12 weeks of CPAP, there is a paucity of data on the effects of NIV or CPAP in patients with OHS. Two small clinical series reported a reduction in pulmonary artery pressure after 3 months or 6 months of NIV therapy only in patients who had echocardiographic evidence of right ventricular overload at baseline. ${ }^{13}{ }^{32}$ In our patients, only NIV reduced the systolic pulmonary artery pressure. We have previously reported that NIV resulted in greater improvements in lung volume, daytime hypercapnia and acid-base balance than $\mathrm{CPAP}^{1}$ (see online only supplementary material). Hypercapnia and acidosis can lead to pulmonary vasoconstriction and an increase in endothelial tone. ${ }^{334}$ Although there were no significant polysomnographic differences at 2 months in the degree of nocturnal hypoxaemia between NIV or CPAP groups, it is possible that patients treated with NIV achieved a better control of nocturnal hypoventilation (in addition to daytime improvement) that may have in turn led to a more significant reduction in pulmonary artery pressures. Although we did not perform transcutaneous $\mathrm{CO}_{2}$ monitoring during sleep, systolic pulmonary artery pressure improvement was correlated with improvements in daytime $\mathrm{PaCO}_{2}$ and $\mathrm{FEV}_{1}$. Moreover, improvement in lung volumes during sleep with NIV can potentially increase the calibre of the airways thereby reducing lower and upper airways resistance which may in turn lead to further improvement of alveolar ventilation. ${ }^{35}$

Although there could be several explanations on how NIV might reduce pulmonary artery pressures, it still remains unclear whether the magnitude of the reduction in the systolic pulmonary artery pressure observed with 2 months of NIV therapy (ie, $3.4 \mathrm{~mm} \mathrm{Hg}$ ) is clinically relevant. Only patients treated with NIV experienced a significant improvement in 6-MWD which may be explained by either reduction in systolic pulmonary artery pressure or by improvement in respiratory function (ie, $\left.\mathrm{FEV}_{1}\right) .{ }^{1}$ Since 6 -MWD improvement was not correlated with the improvement in systolic pulmonary artery pressure, the latter mechanism seems more probable as the causal factor. In a small observational study, it was noted that patients with OHS with right ventricular overload at baseline experienced significant improvements in systolic pulmonary artery pressure and 6-MWD. ${ }^{13}$ Similarly, in our study, the degree of improvement in systolic pulmonary artery pressure and 6-MWD were more robust in the NIV subgroup with systolic pulmonary artery pressure $\geq 40 \mathrm{~mm} \mathrm{Hg}$ at baseline. Moreover, NIV led to a significant decline in the prevalence of pulmonary hypertension. In another study of 278 patients with pulmonary hypertension, standard doses of sildenafil reduced mean pulmonary artery pressure by $2.1 \mathrm{~mm} \mathrm{Hg}$ and led to significant improvement in 6-MWD. ${ }^{36}$ This degree of improvement in mean systolic pulmonary artery pressure is similar to the degree of improvement in pulmonary artery pressure of $3.4 \mathrm{~mm} \mathrm{Hg}$ measured by echocardiography in our study ${ }^{37}$ and lower than the improvement obtained in our patients with pulmonary hypertension at baseline $(6.4 \mathrm{~mm} \mathrm{Hg})$. Although the overall clinical impact of NIV therapy on systolic pulmonary artery pressure in patients with OHS remains unclear, our data reveal that in patients with pulmonary hypertension at baseline, NIV can lead to significant improvement in systolic pulmonary artery pressure and 6-MWD.

In contrast to the limited data in OHS, the impact of CPAP on echocardiographic measures in patients with eucapnic OSA has been assessed in three randomised controlled trials, two observational studies and a case controlled study. ${ }^{30}$ 38-42 Three of these studies assessed the impact of CPAP on measures of LV hypertrophy in patients with OSA and did not find a significant improvement. ${ }^{383943}$ It is important to consider that patients with eucapnic OSA differ significantly from patients with OHS. Indeed, patients with OHS have chronic hypercapnic respiratory failure, experiencing more profound nocturnal hypoxaemia, and have a higher severity of OSA and obesity than patients with eucapnic OSA. Moreover, the prevalence of cardiovascular comorbidities and cardiovascular mortality are significantly higher than patients with eucapnic OSA..$^{9-11}$ Therefore, LV hypertrophy may be more prevalent in patients with OHS and as such, these patients may be more susceptible to derive benefit from short-term NIV therapy. As we have previously reported, NIV is superior to CPAP in improving $\mathrm{FEV}_{1}$ and $6-\mathrm{MWD}^{1}$ (see online supplementary material). We hypothesise that the sustained improvement in acid-base balance, reduction in intrathoracic pressure swings, and improvement in lung function may explain the more robust improvement in LV hypertrophy obtained with short-term NIV than with CPAP therapy.

Our study has several limitations. Although we had described a priori that echocardiography was going to be obtained in all patients enrolled in the Pickwick Study, the original sample size calculation was based on estimated changes in $\mathrm{PaCO}_{2}$, not echocardiographic measures. As such, our sample size may not have been sufficient and therefore increasing the risk of bias. However, we tried to minimise bias by performing adjusted analysis. Another limitation is the short duration of treatment (ie, 2 months). We believe it would have been unethical to extend the period of follow-up beyond 2 months for the control group (ie, lifestyle modification). Accordingly, it is possible that longer duration of therapy would have led to 
more significant improvement in echocardiographic measures. Because the ongoing second phase of the Pickwick Study includes follow-up echocardiographic evaluations, in a future analysis we will be able to compare the short-term and long-term echocardiographic changes between NIV and CPAP. Different echocardiographic equipment and technicians may have increased interobserver variability, although it should have affected all three groups similarly. The absence of nocturnal blood pressure and transcutaneous $\mathrm{CO}_{2}$ monitoring limits our ability to demonstrate that NIV may have been superior at improving ventilation and blood pressure control during sleep. Although we acknowledge the inherent limitations of echocardiography for accurate diagnosis of pulmonary hypertension, it is important to point out that it is a practical and readily available non-invasive tool used routinely in clinical practice. Performing right heart catheterisation in such a large cohort of patients in a clinical trial would not have been pragmatic. Our statistical analysis included multiple group comparisons (ie, NIV, CPAP and control). This may increase the probability of finding statistical significance at random. However, we only performed paired comparisons adjusted by confounders (ANCOVA) if the overall comparison by ANOVA was statistically significant.

In summary, the prevalence of cardiac structural and functional abnormalities as assessed by transthoracic echocardiography is high in patients with OHS. Medium-term NIV therapy seems more effective than CPAP and lifestyle modification in improving measures of LV hypertrophy and systolic pulmonary artery pressure. Moreover, NIV leads to a greater degree of improvement in 6-MWD. Our study suggests that echocardiograms can provide valuable clinical information in the management of patients with OHS being treated with PAP therapy. Long-term comparative studies, however, are necessary to demonstrate whether the beneficial effects of NIV are sustained.

\author{
Author affiliations \\ 'Pneumology Service, San Pedro de Alcantara Hospital, Cáceres, Spain \\ ${ }^{2}$ CIBER de Enfermedades Respiratorias (CIBERES), Madrid, Spain \\ ${ }^{3}$ Cardiology Service, San Pedro de Alcántara Hospital, Cáceres, Spain \\ ${ }^{4}$ Pneumology Service, Virgen del Puerto Hospital, Cáceres, Spain \\ ${ }^{5}$ Pneumology Service, Virgen del Rocío Hospital, Sevilla, Spain \\ ${ }^{6}$ Pneumology Service, University Hospital, Burgos, Spain \\ Pneumology Service, IIS Fundación Jiménez Díaz, Madrid, Spain \\ ${ }^{8}$ Pneumology Service, Hospital Marqués de Valdecilla, Santander, Spain \\ ${ }^{9}$ Pneumology Service, Hospital General Universitario Gregorio Maranon, Madrid, \\ Spain \\ ${ }^{10}$ Pneumology Service, Miguel Servet Hospital, Zaragoza, Spain \\ ${ }^{11}$ Pneumology Service, Hospital Vall d'Hebron, Barcelona, Spain \\ ${ }^{12}$ Pneumology Service, Hospital Universitario 12 de Octubre, Madrid, Spain \\ ${ }^{13}$ Pneumology Service, San Juan Hospital, Alicante, Spain \\ ${ }^{14}$ Sleep Unit and Respiratory Department, Alava University Hospital IRB, Vitoria, Spain \\ ${ }^{15}$ Department of Medicine, University of Chicago, Chicago, Illinois, USA \\ ${ }^{16}$ Department of Medicine, Section of Pulmonary and Critical Care, University of \\ Chicago, Chicago, Illinois, USA
}

Collaborators The Spanish Sleep Network: Rocio Gallego, San Pedro de Alcántara Hospital, Cáceres, Spain, CIBER de enfermedadesrespiratorias (CIBERES), Madrid, Spain; Javier Barca, Extremadura University, Cáceres, Spain; Estrella Ordax, University Hospital, Burgos, Spain, CIBERES, Madrid, Spain; Nicolás González-Mangado, IIS Fundación Jiménez Díaz, Madrid, Spain, (CIBERES), Madrid, Spain; Maria-Ángeles Martinez-Martinez, Valdecilla Hospital, Santander, Spain; Olga Cantalejo, Valdecilla Hospital, Santander, Spain; Elena Ojeda, Gregorio Marañon Hospital, Madrid, Spain; Santiago I Carrizo Miguel, Servet Hospital, Zaragoza, Spain, CIBERES, Madrid, Spain; Begoña Gallego, Miguel Servet Hospital, Zaragoza, Spain; Sergi Martí, Vall d'Hebron Hospital, Barcelona, Spain, CIBERES, Madrid, Spain; Mª Antonia Ramón, Vall d'Hebron Hospital, Barcelona, Spain, CIBERES, Madrid, Spain; Josefa Díaz-de-Atauri, Doce de Octubre Hospital, Madrid, Spain, CIBERES, Madrid, Spain; Jesús MuñozMéndez, Doce de Octubre Hospital, Madrid, Spain, CIBERES, Madrid, Spain; Cristina Senent, San Juan Hospital, Alicante, Spain; Jose N Sancho-Chust, San Juan Hospital, Alicante, Spain; Francisco J Rivas, Alava University Hospital IRB, Vitoria, Spain, CIBERES, Madrid, Spain; Emilia Barrot, Virgen del Rocío Hospital, Sevilla, Spain; José
M Benítez, Virgen de la Macarena Hospital, Sevilla, Spain; Jesús Sanchez-Gómez, Virgen de la Macarena Hospital, Sevilla, Spain; Rafael Golpe, Lucus Augusti University Hospital, Lugo, Spain; AnaSantiago-Recuerda, La Paz Hospital, Madrid, Spain; Silvia Gomez, Arnau de Vilanova Hospital, Lleida, Spain, CIBERES, Madrid, Spain. Mónica Bengoa, University Hospital, Las Palmas, Spain.

Contributors Substantial contributions to study conception and design, acquisition of data, or analysis and interpretation of data: JFM, JC, MVM, BM, MLA-A, Estrella Ordax, Maria F Troncoso, MG, SL-M, JMM, Sergi Marti, TDí-C, EC, CE, Joaquin Teran, NG-M, Angeles Martínez, PDL, Teresa Gomez-Garcia, Santiago J. Carrizo, OR, Maria Antonia Romero PT, CS, MD, Francisco Rivas, AR, CC, Jose Maria Benítez, RG, AS-Recuerda, SG, Monica Bengoa, RG and Maria Angeles Sánchez-Quiroga. Drafting the article or revising the article critically for important intellectual content: JFM; BM, JC; MVM, Roberto M. Lang, Jose M Marin, OR, AR, CC; EC; Joaquin Teran, Maria L Alonso; NG-M; M-Angeles Sánchez-Quiroga MD and PDL. Final approval of the version to be published: JFM, BM, JC; MVM and RML. Agreement to be accountable for all aspects of the work in ensuring that questions related to the accuracy or integrity of any part of the work are appropriately investigated and resolved: JFM. JFM has full access to all data from the study and takes responsibility for the integrity of the data and the accuracy of the data analysis.

Funding Instituto de Salud Carlos III (Fondo de Investigaciones Sanitarias, Ministerio de Sanidad y Consumo) PI050402, Spanish Respiratory Foundation 2005 (FEPAR) and Air Liquide Spain. Funders provided only financial support. They did not participate in the design or conduct of the study, collection, management, analysis, interpretation of the data, preparation, review or approval of the manuscript.

Competing interests None declared.

Ethics approval Comité Ético de Investigación Clínica (CEIC) ID: 28042009.

Provenance and peer review Not commissioned; externally peer reviewed.

(c) Article author(s) (or their employer(s) unless otherwise stated in the text of the article) 2018. All rights reserved. No commercial use is permitted unless otherwise expressly granted.

\section{REFERENCES}

1 Masa JF, Corral J, Alonso ML, et al. Efficacy of different treatment alternatives for obesity hypoventilation syndrome. Pickwick study. Am J Respir Crit Care Med 2015; 192:86-95

2 Sturm R, Hattori A. Morbid obesity rates continue to rise rapidly in the United States. Int J Obes 2013;37:889-91.

3 Balachandran JS, Masa JF, Mokhlesi B. Obesity hypoventilation syndrome epidemiology and diagnosis. Sleep Med Clin 2014;9:341-7.

4 Borel JC, Tamisier R, Gonzalez-Bermejo J, et al. Noninvasive ventilation in mild obesity hypoventilation syndrome: a randomized controlled trial. Chest 2012;141:692-702.

5 Piper AJ, Wang D, Yee BJ, et al. Randomised trial of CPAP vs bilevel support in the treatment of obesity hypoventilation syndrome without severe nocturnal desaturation. Thorax 2008:63:395-401.

6 Masa JF, Corral J, Caballero C, et al. Non-invasive ventilation in obesity hypoventilation syndrome without severe obstructive sleep apnoea. Thorax 2016;71:899-906.

7 Howard ME, Piper AJ, Stevens B, et al. A randomised controlled trial of CPAP versus non-invasive ventilation for initial treatment of obesity hypoventilation syndrome. Thorax 2017;72:437-44.

8 Lévy P, Pépin JL, Arnaud C, et al. Intermittent hypoxia and sleep-disordered breathing: current concepts and perspectives. Eur Respir J 2008;32:1082-95.

9 Castro-Añón O, Pérez de Llano LA, De la Fuente Sánchez S, et al. Obesityhypoventilation syndrome: increased risk of death over sleep apnea syndrome. PLoS One 2015;10:e0117808

10 Basoglu OK, Tasbakan MS. Comparison of clinical characteristics in patients with obesity hypoventilation syndrome and obese obstructive sleep apnea syndrome: a case-control study. Clin Respir J 2014;8:167-74.

11 Priou P, Hamel JF, Person C, et al. Long-term outcome of noninvasive positive pressure ventilation for obesity hypoventilation syndrome. Chest 2010;138:84-90.

12 Nowbar S, Burkart KM, Gonzales R, et al. Obesity-associated hypoventilation in hospitalized patients: prevalence, effects, and outcome. Am J Med 2004;116:1-7.

13 Castro-Añón O, Golpe R, Pérez-de-Llano LA, et al. Haemodynamic effects of noninvasive ventilation in patients with obesity-hypoventilation syndrome. Respirology 2012;17:1269-74

14 Sugerman HJ, Baron PL, Fairman RP, et al. Hemodynamic dysfunction in obesity hypoventilation syndrome and the effects of treatment with surgically induced weight loss. Ann Surg 1988;207:604-13.

15 Kauppert CA, Dvorak I, Kollert F, et al. Pulmonary hypertension in obesityhypoventilation syndrome. Respir Med 2013;107:2061-70.

16 Kessler R, Chaouat A, Schinkewitch P, et al. The obesity-hypoventilation syndrome revisited: a prospective study of 34 consecutive cases. Chest 2001;120:369-76. 
17 Alawami M, Mustafa A, Whyte K, et al. Echocardiographic and electrocardiographic findings in patients with obesity hypoventilation syndrome. Intern Med J 2015;45:68-73.

18 Masa JF, Corral J, Romero A, et al. The Effect of supplemental oxygen in obesity hypoventilation syndrome. J Clin Sleep Med 2016;12:1379-88.

19 Lang RM, Bierig M, Devereux RB, et al. Recommendations for chamber quantification. Eur J Echocardiogr 2006; 7:79-108.

20 Lang RM, Badano LP, Mor-Avi V, et al. Recommendations for cardiac chamber quantification by echocardiography in adults: an update from the American society of echocardiography and the European association of cardiovascular imaging. Eur Heart J Cardiovasc Imaging 2015;16:233-71.

21 Russo V, Di Meo F, Rago A, et al. Impact of continuous positive airway pressure therapy on atrial electromechanical delay in obesity-hypoventilation syndrome patients. J Cardiovasc Electrophysiol 2016;27:327-34.

22 Mancini GB, Dahlöf B, Díez J. Surrogate markers for cardiovascular disease: structural markers. Circulation 2004;109(25 Suppl 1):IV22-20.

23 Zhang Q, Fung JW, Auricchio A, et al. Differential change in left ventricular mass and regional wall thickness after cardiac resynchronization therapy for heart failure. Eur Heart J 2006:27:1423-30.

24 Solomon SD, Appelbaum E, Manning WJ, et al. Effect of the direct Renin inhibitor aliskiren, the Angiotensin receptor blocker losartan, or both on left ventricular mass in patients with hypertension and left ventricular hypertrophy. Circulation 2009;119:530-7.

25 Edelmann F, Wachter R, Schmidt AG, et al. Effect of spironolactone on diastolic function and exercise capacity in patients with heart failure with preserved ejection fraction: the Aldo-DHF randomized controlled trial. JAMA 2013;309:781-91.

26 Klingbeil AU, Schneider M, Martus $P$, et al. A meta-analysis of the effects of treatment on left ventricular mass in essential hypertension. Am J Med 2003;115:41-6.

27 Verdecchia P, Porcellati C, Reboldi G, et al. Left ventricular hypertrophy as an independent predictor of acute cerebrovascular events in essential hypertension. Circulation 2001;104:2039-44.

28 Verdecchia P, Angeli F, Borgioni C, et al. Changes in cardiovascular risk by reduction of left ventricular mass in hypertension: a meta-analysis. Am J Hypertens 2003;16(11 Pt 1):895-9.

29 Fagan KA. Selected Contribution: Pulmonary hypertension in mice following intermittent hypoxia. J App/ Physiol 2001;90:2502-7.

30 Arias MA, García-Río F, Alonso-Fernández A, et al. Pulmonary hypertension in obstructive sleep apnoea: effects of continuous positive airway pressure: a randomized, controlled cross-over study. Eur Heart J 2006;27:1106-13.
31 Sajkov D, Wang T, Saunders NA, et al. Continuous positive airway pressure treatment improves pulmonary hemodynamics in patients with obstructive sleep apnea. Am J Respir Crit Care Med 2002;165:152-8.

32 Held M, Walthelm J, Baron S, et al. Functional impact of pulmonary hypertension due to hypoventilation and changes under noninvasive ventilation. Eur Respir J 2014:43:156-65.

33 Farrukh IS, Gurtner GH, Terry PB, et al. Effect of $\mathrm{pH}$ on pulmonary vascular tone, reactivity, and arachidonate metabolism. J Appl Physiol 1989:67:445-52.

34 Mizuno S, Demura Y, Ameshima S, et al. Alkalosis stimulates endothelial nitric oxide synthase in cultured human pulmonary arterial endothelial cells. Am J Physiol Lung Cell Mol Physiol 2002;283:L113-9.

35 De Backer LA, Vos WG, Salgado R, et al. Functional imaging using computer methods to compare the effect of salbutamol and ipratropium bromide in patient-specific airway models of COPD. Int J Chron Obstruct Pulmon Dis 2011;6:637-46.

36 Galiè N, Ghofrani HA, Torbicki A, et al. Sildenafil Use in Pulmonary Arterial Hypertension (SUPER) Study Group. Sildenafil citrate therapy for pulmonary arterial hypertension. N Engl J Med 2005;353:2148-57.

37 Chemla D, Castelain V, Humbert M, et al. New formula for predicting mean pulmonary artery pressure using systolic pulmonary artery pressure. Chest 2004;126:1313-7.

38 Butt M, Dwivedi G, Shantsila A, et al. Left ventricular systolic and diastolic function in obstructive sleep apnea: impact of continuous positive airway pressure therapy. Circ Heart Fail 2012:5:226-33.

39 Arias MA, García-Río F, Alonso-Fernández A, et al. Obstructive sleep apnea syndrome affects left ventricular diastolic function: effects of nasal continuous positive airway pressure in men. Circulation 2005;112:375-83.

40 Oliveira W, Campos O, Cintra F, et al. Impact of continuous positive airway pressure treatment on left atrial volume and function in patients with obstructive sleep apnoea assessed by real-time three-dimensional echocardiography. Heart 2009;95:1872-8.

41 Akar Bayram N, Ciftci B, Durmaz T, et al. Effects of continuous positive airway pressure therapy on left ventricular function assessed by tissue Doppler imaging in patients with obstructive sleep apnoea syndrome. Eur J Echocardiogr 2009;10:376-82.

42 Dursunoglu N, Dursunoglu D, Ozkurt S, et al. Effects of CPAP on left ventricular structure and myocardial performance index in male patients with obstructive sleep apnoea. Sleep Med 2007:8:51-9.

43 Bilge AR, Yavuz V, Çetin N, et al. The effect of long-term continuous positive airway pressure treatment on systolic and diastolic function in patients with obstructive sleep apnoea syndrome: a five year observational study. Anadolu Kardiyol Derg 2014;14:265-71 